\title{
Inhibition of caspase-1 improves the depressive-like behaviour via regulation of the stability of surface AMPARs
}

\author{
Jian-Guo Chen, Jin-Gang He, Zhuang-Li Hu, Peng-Fei Wu, Li-Hong Long, Fang Wang \\ Tongji Medical College, Huazhong University of Science and Technology, China
}

Background: It is well known that the IL-1beta converting enzyme caspase- 1 is an inflammatory caspase, and activation of caspase- 1 involves the response of immune cells to both pathogen-derived and endogenous mediators by the formation of inflammasome complexes. In addition to its role in peripheral inflammation, growing evidences also indicate that caspase -1 mediated neuroinflammation in the brain mediates depression-like behavior induced by LPS or chronic stress. However, how the increased caspase-1 in the brain influences the depressive-like behavior remains largely unknown. Methods: To address this issue, we examined the effects and underlying mechanisms of caspase- 1 on preclinical murine models of depression. Results: We found that loss of caspase-1 expression in caspase- 1 knockout mice alleviated chronic stress-induced depression-like behaviors, whereas overexpression of caspase- 1 in the hippocampus of wild-type mice was sufficient to induce depression-like behaviors. Chronic social defeat stress (CSDS) reduced glutamatergic neurotransmission and decreased surface expression of AMPA receptors in hippocampal neurons of WT mice, but not caspase-1 knockout mice. Importantly, pharmacological inhibition of caspase-1 signaling pathway prevented the depression-like behaviors and the decrease in surface expression of AMPARs in stressed WT mice. CSDS-induced depression-like behaviors can be mimicked by exogenous intracerebroventricular administration of IL-1beta in both WT and caspase-1 knockout mice. Conclusion: Our findings demonstrate that an increase in the caspase- 1 facilitates AMPAR internalization in the hippocampus, which dysregulates glutamatergic synaptic transmission, eventually resulting in depression-like behaviors. 